\title{
Ensino Remoto Emergencial: Implantação e resultados na percepção de estudantes universitários
}

\author{
Emergency Remote Teaching: Implementation and results in the perception of university students \\ Enseñanza Remota de Emergencia: Implementación y resultados en la percepción de estudiantes \\ universitarios
}

Recebido: 30/06/2021 | Revisado: 07/07/2021 | Aceito: 12/07/2021 | Publicado: 22/07/2021

\author{
Maria Luiza Ferreira Stringhini \\ ORCID: https://orcid.org/0000-0003-2012-7677 \\ Universidade Federal de Goiás, Brasil \\ E-mail: mlstring@ufg.br \\ Ida Helena Carvalho Francescantonio Menezes \\ ORCID: https://orcid.org/0000-0003-4214-7206 \\ Universidade Federal de Goiás, Brasil \\ E-mail: fmenezes@ufg.br \\ Débora Danielle Alves Moraes Priebe \\ ORCID: https://orcid.org/0000-0002-8979-4331 \\ Universidade Federal de Goiás, Brasil \\ E-mail: deboradanielle@ufg.br \\ Rodrigo Barbosa Monteiro Cavalcante \\ ORCID: https://orcid.org/0000-0003-2649-6588 \\ Universidade Federal de Goiás, Brasil \\ E-mail: rodrigobarbosa@ufg.br \\ Ana Tereza Vaz de Souza Freitas \\ ORCID: https://orcid.org/0000-0002-6785-3433 \\ Universidade Federal de Goiás, Brasil \\ E-mail: anaterezavaz@ufg.br \\ Marilia Mendonça Guimarães \\ ORCID: https://orcid.org/0000-0003-1466-6180 \\ Universidade Federal de Goiás, Brasil \\ E-mail: marilianutri@ufg.br \\ Thaísa Anders Carvalho Souza \\ ORCID: https://orcid.org/0000-0002-5851-1343 \\ Universidade Federal de Goiás, Brasil \\ E-mail: thaisa_anders@ufg.br \\ Tânia Aparecida Pinto de Castro Ferreira \\ ORCID: https://orcid.org/0000-0003-2472-5025 \\ Universidade Federal de Goiás, Brasil \\ E-mail: taniaferreira@ufg.br \\ Karine Anusca Martins \\ ORCID: https://orcid.org/0000-0003-4992-4522 \\ Universidade Federal de Goiás, Brasil \\ E-mail: karine_anusca@ufg.br \\ Andréa Sugai Mortoza \\ ORCID: https://orcid.org/0000-0002-2185-9143 \\ Universidade Federal de Goiás, Brasil \\ E-mail: andreasugai@ufg.br
}

\begin{abstract}
Resumo
O objetivo do trabalho foi avaliar a percepção dos estudantes de um Curso de Nutrição de uma Universidade Pública do Brasil ao Ensino Remoto Emergencial (ERE). Foi realizado um estudo transversal em dois momentos, no início e após implementação do ERE, considerando-se os alunos segundo sistema de acesso à Universidade, por meio de Ações Afirmativas (AA) ou Ampla Concorrência (AC). Inicialmente, participaram 351 ingressos, 159 por AA e 192 por AC, via Google Forms, com o intuito de compreender dificuldades e facilidades em relação à implementação do ERE. No segundo momento, foi aplicado um formulário eletrônico atitudinal do tipo Likert, contemplando aspectos pedagógicos e de ambiente de estudo. A comparação entre os escores dos estudantes ingressos por AA e AC foi realizada pelo teste $\mathrm{t}$ de Student. Os dados provenientes do formulário foram apresentados em medianas e intervalosinterquartis e as variáveis categóricas, expressas em frequências absolutas e relativas. A associação entre as variáveis categóricas foi verificada pelo teste qui-quadrado de independência. O nível de significância foi de 5\%. Indivíduos AA apresentaram dificuldades significativas de acesso e do sinal de Internet, na aquisição de equipamentos e pior
\end{abstract}


qualidade do ambiente de estudo no domicílio. Com o auxílio institucional, os estudantes julgaram que o ERE foi necessário e atendeu as exigências educacionais advindas da pandemia no primeiro semestre de implantação. A continuidade do ERE irá demandar, por parte das gestões das Unidades Acadêmicas, monitoramento dos aspectos acadêmicos e resolução das dificuldades junto às demais instâncias envolvidas com o ensino superior.

Palavras-chave: Ensino superior; Aprendizagem; Política governamental; Desigualdade social.

\begin{abstract}
The aim of this work was to evaluate the perception of students from a Nutrition Course at a Public University in Brazil to the Emergency Remote Teaching. A cross-sectional study was carried out in two moments, at the beginning and after implementation of the Emergency Remote Teaching, considering the students according to the University access system, through public policies of Affirmative Actions (AA) or Broad Competition (BC). Initially, 159 entries per AA and 192, per BC, via Google Forms participated, in order to understand the difficulties and facilities in relation to the implementation of the Emergency Remote Teaching. In the second moment, an attitudinal electronic form of the Likert type was applied, covering pedagogical aspects and study environment. The comparison between the scores of the AA and BC students was performed using Student's t test. Likert-type items were presented as medians and interquartile ranges, and categorical variables, expressed as absolute and relative frequencies. The association between categorical variables was verified by the chi-square test of independence. The significance level was 5\%. AA individuals had significant difficulties in accessing, signal and equipment quality, and also presented poorer quality of the study environment at home. With institutional help, the students judged that the Emergency Remote Teaching was necessary and met the educational requirements arising from the pandemic in the first semester of implementation. Continuing Emergency Remote Teaching will require, on the part of the management of Academic Units, monitoring of academic aspects and resolution of difficulties with other instances involved with higher education.
\end{abstract}

Keywords: Higher education; Learning; Social policy; Social inequality.

\title{
Resumen
}

El objetivo de este estudio fue evaluar la percepción de los estudiantes de un Curso de Nutrición en una Universidad Pública de Brasil hacia el Enseñanza Remota de Emergencia (ERE). Se realizó un estudio transversal en dos momentos, al inicio y posterior a la implementación del ERE, considerando a los estudiantes según el sistema de acceso a la Universidad, a través de Acciones Afirmativas (AA) o Competencia Amplia (CA). Inicialmente participaron 351 entradas, 159 de AA y 192 de CA, a través de Google Forms, con el fin de comprender las dificultades y facilidades en relación a la implementación del ERE. En el segundo momento, se aplicó una forma electrónica actitudinal tipo Likert, cubriendo aspectos pedagógicos y ambiente de estudio. La comparación entre las puntuaciones de los estudiantes matriculados en AA y CA se realizó mediante la prueba t de Student. Los datos del formulario se presentaron como medianas y rangos intercuartílicos, y las variables categóricas, expresadas como frecuencias absolutas y relativas. La asociación entre variables categóricas se verificó mediante la prueba de independencia de chi-cuadrado. El nivel de significancia fue del 5\%. Los individuos de AA tenían dificultades significativas en el acceso, la calidad de la señal, la adquisición de equipos y una peor calidad del entorno de estudio en el hogar. Con ayuda institucional, los estudiantes juzgaron que el ERE era necesario y cumplió con los requisitos educativos derivados de la pandemia en el primer semestre de implementación. La Educación Remota de Emergencia Continuada requerirá, por parte de la dirección de las Unidades Académicas, el seguimiento de los aspectos académicos y la resolución de dificultades con otras instancias relacionadas con la educación superior.

Palabras clave: Enseñanza superior; Aprendizaje; Política gubernamental; Desigualdad social.

\section{Introdução}

Com o advento da pandemia mundial pelo Sars-CoV-2 (Covid-19) medidas de isolamento social, uso de máscaras e higienização constante das mãos foram necessárias para controlar a disseminação do vírus. A implantação de ações de enfrentamento da pandemia, como a suspensão de atividades de ensino presencial nas Instituições de Ensino Superior (IES), foi considerada estratégia fundamental para não sobrecarregar o sistema de saúde no mundo e no Brasil e garantir a integridade física de estudantes e servidores (Al Samaraee, 2020; Sandhu \& de Wolf, 2020). Nestas condições, em março de 2020, a Universidade Federal de Goiás (UFG), Goiânia, Goiás, Brasil, por meio do seu Conselho Universitário (CONSUNI), após discussões com as unidades acadêmicas e os Núcleos Docentes Estruturantes (NDE) decidiu pela suspensão das aulas presenciais e adoção do Ensino Remoto Emergencial (ERE). Essa decisão também ocorreu em diversas instituições de ensino do mundo em todos os níveis, no básico (Cardoso, 2020), no médio (Tanik-Önal \& Önal, 2020) e no superior (Joaquin et al.,

2020; Sindiani et al., 2020). 
O processo decisório da UFG passou por avaliação das possibilidades de implantação do chamado "Ensino Mediado por Tecnologias de Informação e Comunicação Digitais” (TDIC). O então conhecido Ensino Remoto Emergencial (ERE), que difere da educação à distância, demandou ajustes no sentido da UFG garantir o acesso a todo e qualquer estudante matriculado na universidade, independente da forma de ingresso, fosse por ampla concorrência (AC), UFGInclui ou ações afirmativas (AA).

A distinção quanto a forma de ingresso faz-se necessária uma vez que as vagas oferecidas por AA visam atuar como uma ação política reparatória, compensatória ou preventiva, que busca a valorização social, econômica, política e cultural, a depender do contexto histórico e social do grupo considerado em vulnerabilidades (Moehlecke, 2002). Assim, a partir da Lei $\mathrm{n}^{\circ}$ 12.711/2012, as universidades federais destinam vagas para os estudantes que cursaram integralmente o ensino médio em escolas públicas. A reserva de, no mínimo 50\% das vagas, encontra-se subdividida em dois grupos: para os estudantes oriundos de famílias com renda per capita mensal menor que 1,5 salário-mínimo e para os estudantes oriundos de famílias com renda per capita mensal maior que 1,5 salário-mínimo. Para estes dois grupos há um subgrupo com reserva de vagas para os estudantes autodeclarados pretos, pardos, indígenas (PPI) e para as pessoas com deficiência. O número de vagas é proporcional ao quantitativo populacional da unidade federativa onde está instalada a instituição (Brasil, 2012). Apesar da perspectiva da garantia de acesso ao ensino, esse grupo de estudantes apresenta dificuldades quanto à permanência, devendo ser sistematicamente monitorado para o cumprimento da finalização no ensino superior (Fórum Nacional de Pró- Reitores de Assuntos Comunitários e Estudantis, 2019). Os demais alunos dão entrada na universidade por Ampla Concorrência (AC).

Para suprir os alunos de recursos para continuarem seu processo de ensino aprendizagem, se desenvolveu várias ações como a publicação de editais de doação de equipamentos de informática e chips de internet, dentre outras. A Universidade ofereceu duas plataformas online para repositório de conteúdo e administração das rotinas pedagógicas, para além do Sistema Integrado de Gestão de Atividades Acadêmicas (SIGAA), como o Moodle e o Google Classroom (Universidade Federal de Goiás [UFG], 2020a).

No segundo semestre de 2020, continuando o ensino no formato ERE, novos desafios e lacunas foram enfrentados. Outros processos de trabalho foram demandados, quanto à administração online, metodologias de ensino, capacitação docente, e atividades de adaptações do domicílio, no confronto com a inovação tecnológica. Soma-se a isso, necessitou-se de adequação de docentes, técnicos e estudantes às novas rotinas e horários, com a reorganização do calendário acadêmico. Além da preocupação do acesso, principalmente dos discentes, às tecnologias de informação e comunicação, houve a apreensão quanto à saúde mental de toda comunidade universitária e familiar (Rajab et al., 2020; Universidade Federal de Goiás [UFG], 2020b; Universidade Federal de Goiás [UFG], 2020c).

Ainda são desconhecidas as consequências no sistema de educação brasileiro, quiçá mundial, diante de todas as mudanças necessárias para atender as demandas provocadas pela pandemia de Covid-19. Diversos questionamentos continuarão sendo formulados e a avaliação contínua de toda essa abordagem é premente, especialmente, no que se diz respeito às TDIC, tão abruptamente inserida no processo de ensino, e que com certeza, vai fazer e continuará fazendo parte do campo de aprendizagem futuro, caracterizando o formato híbrido (Pérez-López et al., 2021).

Diante do exposto, o objetivo deste estudo foi verificar a percepção dos estudantes de Nutrição de uma universidade pública brasileira quanto a implementação do ERE durante o período da pandemia de Covid-19 e suspensão das aulas presenciais, como forma de acompanhamento contínuo e subsídio de novas ações na formação acadêmica dos estudantes.

\section{Metodologia}

O estudo faz parte do projeto de avaliação do Projeto Pedagógico do Curso (PPC) de Nutrição, cadastrado no Comitê de Ética em Pesquisa (CEP) da UFG sob número de processo 4.491.078. 
Paralelamente às diversas ações institucionais, o NDE da Faculdade de Nutrição (FANUT) realizou um estudo transversal com todos os estudantes regularmente matriculados no curso $(n=370)$ em dois momentos distintos. O primeiro momento levou em consideração a heterogeneidade no formato do ingresso pelo estudante: por Ações Afirmativas (AA) ou por Ampla Concorrência (AC). Assim, em junho de 2020, participaram 351 (94,8\%), sendo 159 ingressos por Ações Afirmativas (AA) e 192 ingressos por Ampla Concorrência (AC). A amostra obtida atende a um nível de confiança de 99\%, com margem de erro amostral de 1,56\%. Foi realizado um levantamento das dificuldades e facilidades destes em relação a implementação do ERE. Aplicou-se um questionário, via Google Forms, com 13 questões de múltipla escolha, enviado por meio do e-mail institucional e do grupo de WhatsApp composto por estudantes e Coordenação do Curso.

Este questionário compreendeu aspectos relacionados à conectividade de internet: tipo de acesso, qualidade do sinal, equipamentos disponíveis (tipo de equipamento, se compartilha com outras pessoas da residência e tempo disponível para utilização) e acesso ao pacote Microsoft Office. Outras questões relacionadas à qualidade do ambiente de estudo, além de quais atividades remotas seriam capazes de realizar, ferramentas aptas, domínio das TDIC e necessidade de apoio institucional para realizar atividades remotas também constavam no questionário. Ao final, ofereceu-se a possibilidade de comentários gerais em relação ao tema. O resultado subsidiou a coordenação do curso de Nutrição no desenvolvimento de ações, em parceria com o NDE, para facilitar o acesso dos estudantes ao ERE.

As questões referentes à qualidade da internet, qualidade do ambiente de estudos e domínio em relação ao uso das TDIC foram compostas por uma escala de onze pontos, sendo 0 para péssimo e 10 para excelente. Posteriormente os valores foram classificados em três faixas, sendo de 0 a 6 pontos considerado como ruim, 7 como regular e de 8 a 10 como satisfatório.

Em um segundo momento, após o primeiro semestre de implantação do ERE (janeiro de 2021), foi aplicado um instrumento atitudinal do tipo Likert, utilizando-se formulário eletrônico Google Forms por meio da plataforma GSuite, a todos os estudantes do $1^{\circ}$ ao $8^{\circ}$ período do curso de Nutrição $(\mathrm{n}=321)$. O uso da escala Likert teve como finalidade evidenciar a relevância atribuída às questões propostas acerca de diversos aspectos do ERE. Responderam ao questionário 93 estudantes (28,9\%), sendo 43 ingressos por AA e 50 por AC. A amostra obtida nessa etapa atende a um nível de confiança de $95 \%$, com margem de erro amostral de 8,58\%. O instrumento foi composto por assertivas positivas e negativas distribuídas em duas dimensões: D1) Aspectos Pedagógicos e D2) Ambiência (Quadro 1). Para a dimensão Aspectos Pedagógicos foram observados os materiais de apoio pedagógico fornecidos pelos professores, os métodos e instrumentos de avaliação, a participação e a aprendizagem dos discentes, o aproveitamento, a satisfação e pertinência das metodologias utilizadas. Para a dimensão Ambiência, foi observado o ambiente de estudo (condições e rotinas de estudo), a conectividade e disponibilidade de equipamentos, e a interação entre discentes e docentes.

Quadro 1. Assertivas da escala Likert referentes às dimensões do Ensino Remoto Emergencial do Curso de Graduação em Nutrição, UFG. Goiânia, Goiás, Brasil, 2020

\begin{tabular}{|c|c|c|c|}
\hline Dimensões & Assertivas & $\begin{array}{c}\text { Escore final } \\
\text { mínimo }\end{array}$ & $\begin{array}{c}\text { Escore final } \\
\text { máximo }\end{array}$ \\
\hline Aspectos pedagógicos & $2,3,9,10,12,13,14,15,16,17$ & 10 & 40 \\
\hline Ambiência & $1,4,5,6,7,8,11,18$ & 8 & 32 \\
\hline
\end{tabular}

Fonte: Autores.

As assertivas foram pontuadas em escalas de quatro pontos que variaram de 1 a 4 , associadas a uma escala atitudinal de discordância plena (DP) a concordância plena (CP), com os seguintes termos intermediários: inclinado a discordar (ID) e inclinado a concordar (IC). Em seguida, as assertivas foram randomizadas para a aplicação do instrumento. As percepções do 
público-alvo foram interpretadas baseando-se na média do somatório da pontuação obtida pelos discentes em cada dimensão, sendo, na dimensão Aspectos Pedagógicos: de 10 a 19,9 pontos uma percepção insatisfatória, de 20 a 29,9 pontos uma relativa satisfação e de 30 a 40 pontos uma alta satisfação; e na dimensão Ambiência: de 8 a 15,9 pontos uma percepção insatisfatória, de 16 a 23,9 pontos uma relativa satisfação e de 24 a 32 pontos uma alta satisfação. Na análise individual dos dezoitos itens tipo-Likert foram consideradas as medianas, sendo de 1,00 a 1,99 pontos uma percepção insatisfatória, mostrando a necessidade de uma intervenção em curto prazo; de 2,00 a 2,99 pontos uma relativa satisfação, revelando aspectos a serem melhorados e exigindo medidas de médio prazo; e de 3,00 a 4,00 pontos uma alta satisfação.

A percepção dos discentes com o ERE foi mensurada pelo escore obtido no somatório dos itens da escala Likert. A confiabilidade interna das questões e das duas dimensões foi verificada pelo coeficiente alfa de Cronbach. O referido coeficiente indica a concordância e consistência interna dos itens de um instrumento de coleta de dados, variando de 0 (ausência de confiabilidade) a 1 (confiabilidade perfeita). Segundo Hair et al. (2002) são considerados aceitáveis os valores de alfa maiores que $0,70 . \mathrm{Na}$ análise de confiabilidade interna das dezoito assertivas Likert do questionário obteve-se o coeficiente alfa de Cronbach de 0,90. Na dimensão Aspectos pedagógicos, composta por dez assertivas, tal coeficiente foi de 0,89 , e na dimensão Ambiência, formada por oito assertivas, o valor obtido foi de 0,70. Valores altos de coeficiente $\alpha>0,90$ são considerados coeficientes satisfatórios. Os valores dos coeficientes do presente estudo demonstraram a consistência interna do questionário e de suas dimensões (Hair et al., 2002).

Ao final do formulário, havia quatro perguntas de múltipla escolha quanto às dinâmicas de atividades utilizadas para as aulas teóricas e práticas, a distribuição da carga horária das disciplinas em atividades síncronas e assíncronas e quanto às plataformas de TDIC como ambiente de ensino/aprendizagem, além de um espaço para comentários. Todos os participantes assinaram o termo de consentimento livre e esclarecido (TCLE) com as informações sobre as etapas do estudo.

A comparação entre os escores dos estudantes ingressos por AA e AC foi realizada pelo teste $\mathrm{t}$ de Student. Os itens tipo-Likert foram apresentados em medianas e intervalos-interquartis (25\%-75\%), considerando sua natureza ordinal e as variáveis categóricas foram apresentadas em frequências absolutas e relativas. A existência de associação entre as variáveis categóricas foi verificada pelo teste qui-quadrado de independência. O nível de significância considerado em todas as análises foi de 5\%. Os resultados foram compilados em planilhas do Microsoft Office Excel e analisados no pacote estatístico IBM SPSS versão 20.

\section{Resultados}

Condições de conectividade e ambiente de estudo dos estudantes para iniciar o Ensino Remoto Emergencial

Os dados do primeiro momento do estudo permitiram caracterizar a conectividade e as condições de ambiência de estudo. A principal forma de acesso à internet foi por meio da banda larga (cabo ou Wi-fi). Quase metade dos participantes também tinha acesso a dados móveis $(4 \mathrm{G})$. Entretanto, a forma de acesso diferiu entre aqueles ingressos por AA e aqueles por $\mathrm{AC}(\mathrm{p}<0,001)$, de modo que o acesso concomitante à banda larga e aos dados móveis se deu entre a maioria dos estudantes AC $(\mathrm{n}=101 ; 52,6 \%)$, enquanto o acesso unicamente à banda larga foi apontado pela maior parte dos AA ( $\mathrm{n}=104 ; 65,4 \%)$ (Tabela $1)$.

Um terço dos participantes ( $\mathrm{n}=104 ; 29,6 \%)$ informa ter acesso a uma qualidade de sinal não ideal ao desenvolvimento das atividades remotas, sendo também verificada diferença significativa entre as categorias de ingresso, tendo melhor acesso quem ingressou por AC. Dentre os equipamentos que poderiam ser utilizados no ERE, todos afirmaram possuir pelo menos um dos dispositivos listados no formulário de coleta de dados (notebook, computador de mesa, celular ou tablet). A maior parte ( $\mathrm{n}=266 ; 75,8 \%$ ) possui, concomitantemente, computador (desktop ou notebook) e celular (e/ou tablet), seguida daqueles que possuem apenas celular e/ou tablet $(n=57 ; 16,2 \%)$. Entretanto, $113(32,19 \%)$ estudantes informaram dividir a utilização do 
equipamento e, desses, $36(31,8 \%)$ tinham a disponibilidade máxima de uso de até duas horas por dia. A maioria informou ter acesso ao pacote Office $(\mathrm{n}=281 ; 80,1 \%)$. Para mais de um terço $(\mathrm{n}=129 ; 36,8 \%)$ o ambiente domiciliar não foi favorável ao estudo remoto, diferindo significativamente entre os estudantes AA e AC (Tabela 1).

Os principais apoios institucionais necessários para o desenvolvimento da ERE foram: ambiente de estudo ( $\mathrm{n}=85$; $24,2 \%)$, equipamentos $(n=79 ; 22,5 \%)$, capacitação técnica $(n=57 ; 16,2 \%)$, acesso ou conexão à internet $(n=50 ; 14,2 \%)$. Os estudantes ingressos pelas ações afirmativas necessitavam mais de equipamentos $(p=0,002)$ e melhor ambiente de estudo ( $p$ $<0,0001)$ quando comparados com os estudantes ingressos por AC. A prevalência da não necessidade de apoio da instituição foi maior entre os alunos ingressos pelas AC ( $\mathrm{p}<0,0001)$. Entre as atividades possíveis de realização, diante das condições dos equipamentos e acesso à internet destacaram-se: assistir a videoaulas gravadas ( $\mathrm{n}=327 ; 93,1 \%)$, ler textos teóricos sugeridos pelo professor ( $\mathrm{n}=322 ; 91,7 \%)$ e fazer listas de atividades enviadas pelo professor $(\mathrm{n}=318 ; 90,5 \%)$, sendo a plataforma Moodle e o $B \log$ as menos referidas ( $\mathrm{n}=96 ; 27,3 \%$ e $\mathrm{n}=74 ; 21,1 \%$, respectivamente). $\mathrm{O}$ e-mail ( $\mathrm{n}=317 ; 90,3 \%)$, as videoaulas gravadas ( $\mathrm{n}=315 ; 89,7 \%)$ e o Sistema Integrado de Gestão de Atividades Acadêmicas (SIGAA) ( $\mathrm{n}=300 ; 85,5 \%)$ foram as ferramentas de TDIC que os estudantes informaram possuir mais aptidão para a utilização (Tabela 1).

Diferenças significativas foram encontradas no quantitativo de ingressos por AA e AC quanto à possibilidade de participação de aulas e reuniões por videoconferência, avaliações e palestras online. Com relação às ferramentas que são aptos a usar, a diferença entre as duas categorias foi observada nas plataformas de videoconferência, Instagram, YouTube e Blog.

Tabela 1. Condições de conectividade e ambiente de estudo dos estudantes ao iniciarem o Ensino Remoto Emergencial (ERE). Goiânia, Goiás, Brasil, 2020.

\begin{tabular}{|c|c|c|c|c|c|}
\hline \multirow[t]{2}{*}{ Questionamentos } & & AA & $\mathrm{AC}$ & Total & Valor-p* \\
\hline & & $159(45,3 \%)$ & $192(54,7 \%)$ & $351(100 \%)$ & \\
\hline \multirow[t]{4}{*}{ Acesso à internet } & Banda larga (cabo ou Wi-fi) e dados móveis & $40(11,4)$ & $101(28,8)$ & $141(40,2)$ & \\
\hline & & & & & $<0,001$ \\
\hline & Apenas banda larga (cabo ou Wi-fi) & $104(29,6)$ & $84(23,9)$ & $188(53,6)$ & \\
\hline & $\begin{array}{c}\text { Apenas dados móveis (4G) ou não possui } \\
\text { internet }\end{array}$ & $15(4,3)$ & $7(2,0)$ & $22(6,3)$ & \\
\hline \multirow{3}{*}{$\begin{array}{l}\text { Qualidade do sinal } \\
\text { de internet }\end{array}$} & $0-6$ & $61(17,4)$ & $43(12,3)$ & $104(29,6)$ & $\mathbf{0 , 0 3}$ \\
\hline & 7 & $37(10,5)$ & $46(13,1)$ & $83(23,6)$ & \\
\hline & $8-10$ & $61(17,4)$ & $103(29,3)$ & $164(46,7)$ & \\
\hline \multirow{4}{*}{$\begin{array}{l}\text { Equipamentos de } \\
\text { acesso à internet }\end{array}$} & Computador (desktop ou notebook) e & $117(33,3)$ & $149(42,5)$ & $266(75,8)$ & \\
\hline & celular (ou tablet) & & & & 0,018 \\
\hline & Apenas computador (desktop ou notebook) & $8(2,3)$ & $20(5,7)$ & $28(8,0)$ & \\
\hline & Apenas celular (ou tablet) & $34(9,7)$ & $23(6,6)$ & $57(16,2)$ & \\
\hline \multirow{4}{*}{$\begin{array}{c}\text { Equipamento } \\
\text { compartilhado e } \\
\text { tempo disponível } \\
\text { para uso }\end{array}$} & Não, apenas eu uso & $104(29,6)$ & $134(38,2)$ & $238(67,8)$ & 0,127 \\
\hline & Sim, tenho mais de $2 \mathrm{~h} /$ dia para uso & $32(9,1)$ & $45(12,8)$ & $77(21,9)$ & \\
\hline & Sim, tenho de $1 \mathrm{~h}$ a $2 \mathrm{~h} /$ dia para uso & $18(5,1)$ & $10(2,8)$ & $28(8,0)$ & \\
\hline & Sim, tenho menos de $1 \mathrm{~h} /$ dia para uso & $5(1,4)$ & $3(0,9)$ & $8(2,3)$ & \\
\hline \multirow{3}{*}{$\begin{array}{l}\text { Acesso ao pacote } \\
\text { Office no } \\
\text { computador ou } \\
\text { celular }\end{array}$} & Sim & $121(34,5)$ & $160(45,6)$ & $281(80,1)$ & \\
\hline & & & & & 0,091 \\
\hline & Não & $38(10,8)$ & $32(9,1)$ & $70(19,9)$ & \\
\hline \multirow{3}{*}{$\begin{array}{c}\text { Qualidade do } \\
\text { ambiente de estudo } \\
\text { em casa }\end{array}$} & $0-6$ & $82(23,4)$ & $47(13,4)$ & $129(36,8)$ & \\
\hline & 7 & $26(7,4)$ & $34(9,7)$ & $60(17,1)$ & $<0,001$ \\
\hline & $8-10$ & $51(14,5)$ & $111(31,6)$ & $162(46,2)$ & \\
\hline
\end{tabular}


Research, Society and Development, v. 10, n. 9, e11610917744, 2021

(CC BY 4.0) | ISSN 2525-3409 | DOI: http://dx.doi.org/10.33448/rsd-v10i9.17744

\begin{tabular}{|c|c|c|c|c|c|}
\hline Domínio em relação & $0-6$ & $62(17,7)$ & $60(17,1)$ & $122(34,8)$ & \\
\hline ao uso das & & & & & 0,310 \\
\hline $\begin{array}{l}\text { Tecnologias Digitais } \\
\text { de Informação e }\end{array}$ & 7 & $34(9,7)$ & $48(13,7)$ & $82(23,4)$ & \\
\hline $\begin{array}{l}\text { Comunicação } \\
\text { (TDIC) }\end{array}$ & $8-10$ & $63(17,9)$ & $84(23,9)$ & $147(41,9)$ & \\
\hline \multirow{5}{*}{$\begin{array}{c}\text { Necessidade de } \\
\text { apoio institucional } \\
\text { para realizar as } \\
\text { atividades remotas }\end{array}$} & Equipamentos & $48(13,6)$ & $31(8,8)$ & $79(22,5)$ & 0,002 \\
\hline & Acesso / conexão de internet & $26(7,4)$ & $24(6,8)$ & $50(14,2)$ & 0,304 \\
\hline & Capacitação técnica & $29(8,2)$ & $28(8,0)$ & $57(16,2)$ & 0,355 \\
\hline & Ambiente de estudo & $59(16,8)$ & $26(4,4)$ & $85(24,2)$ & $<0,001$ \\
\hline & Nenhum & $68(19,3)$ & $125(35,6)$ & $193(54,9)$ & $<0,001$ \\
\hline \multirow{9}{*}{$\begin{array}{l}\text { Atividades possíveis } \\
\text { de realizar } \\
\text { considerando o } \\
\text { acesso à internet e os } \\
\text { equipamentos } \\
\text { disponíveis }^{\mathrm{a}}\end{array}$} & Assistir a videoaulas (gravadas) & $145(41,3)$ & $182(51,8)$ & $327(93,1)$ & 0,183 \\
\hline & Participar de aulas por videoconferência & $69(19,6)$ & $139(39,6)$ & $208(59,2)$ & $<0,001$ \\
\hline & Ler textos teóricos sugeridos pelo professor & $142(40,4)$ & $180(51,3)$ & $322(91,7)$ & 0,132 \\
\hline & $\begin{array}{c}\text { Fazer listas de atividade enviadas pelo } \\
\text { professor }\end{array}$ & $142(40,4)$ & $176(50,1)$ & $318(90,5)$ & 0,451 \\
\hline & Realizar avaliações online & $95(27,1)$ & $149(42,4)$ & $244(69,5)$ & $<0,001$ \\
\hline & Assistir palestras online & $95(27,1)$ & $150(42,7)$ & $245(69,8)$ & $<0,001$ \\
\hline & Utilizar o ambiente Moodle & $60(17,1)$ & $79(22,5)$ & $139(39,6)$ & 0,515 \\
\hline & Realizar atividades complementares & $108(30,7)$ & $132(37,6)$ & $240(68,3)$ & 0,868 \\
\hline & Participar de reuniões por videoconferência & $65(18,5)$ & $129(36,7)$ & $194(55,2)$ & $<0,001$ \\
\hline \multirow{13}{*}{$\begin{array}{l}\text { Ferramentas de } \\
\text { TDIC que considera } \\
\text { ter aptidão para uso }\end{array}$} & $\begin{array}{c}\text { SIGAA - Sistema Integrado de Gestão } \\
\text { Acadêmica }\end{array}$ & $133(37,9)$ & $167(47,6)$ & $300(85,5)$ & 0,378 \\
\hline & E-mail & $143(40,7)$ & $174(49,6)$ & $317(90,3)$ & 0,828 \\
\hline & Moodle & $38(10,8)$ & $58(16,5)$ & $96(27,3)$ & 0,187 \\
\hline & Videoaulas (gravadas) & $142(40,4)$ & $173(49,3)$ & $315(89,7)$ & 0,806 \\
\hline & Videoconferências (ao vivo) & $53(15,1)$ & $122(34,7)$ & $175(49,8)$ & $<0,001$ \\
\hline & Drive de armazenamento e & $112(31,9)$ & $146(41,6)$ & $258(73,5)$ & 0,236 \\
\hline & $\begin{array}{c}\text { compartilhamento de dados (ex. Google } \\
\text { Drive) }\end{array}$ & & & & \\
\hline & Facebook & $49(14,0)$ & $73(20,7)$ & $122(34,7)$ & 0,158 \\
\hline & Instagram & $94(26,8)$ & $137(39,0)$ & $231(65,1)$ & 0,0162 \\
\hline & Whatsapp & $124(35,3)$ & $155(44,2)$ & $279(79,5)$ & 0,526 \\
\hline & Youtube & $104(29,6)$ & $153(43,6)$ & $257(73,2)$ & 0,0026 \\
\hline & $\mathrm{B} \log$ & $19(5,4)$ & $55(15,7)$ & $74(21,1)$ & $<0,001$ \\
\hline & Nenhum & $1(0,2)$ & $0(0,0)$ & $1(0,2)$ & - \\
\hline
\end{tabular}

Nota. Tabela com percepções dos estudantes acerca da experiência vivenciada durante o primeiro semestre de implantação do ERE. AA= discentes ingressantes no ensino superior por Ações Afirmativas; $\mathrm{AC}=$ discentes ingressantes por Ampla Concorrência.

${ }^{a}$ Questões com possibilidade de seleção de mais de uma alternativa.

* Qui-quadrado de independência com 005 de significância (probabilidade de erro de 5\%).

Fonte: Autores.

Na segunda etapa do estudo 93 estudantes do $1^{\circ}$ ao $8^{\circ}$ período do curso preencheram o questionário, sendo 43 AA e 50 AC. A comparação da média dos escores finais obtidos pelos estudantes ingressos por AA e AC nas duas dimensões do questionário encontra-se na Tabela 2. 
Tabela 2. Escores finais, em médias e desvios-padrão, obtidos nas dimensões Pedagógicas e de Ambiência. Goiânia, Goiás, Brasil, 2020.

\begin{tabular}{lcccc}
\hline Dimensões & $\mathbf{A A}$ & $\mathbf{A C}$ & Total & Valor-p* \\
& $\mathbf{n = 4 3}$ & $\mathbf{n = 9 5 0}$ & \\
\hline Pedagógica & $28,07(\mathrm{DP}=6,03)$ & $27,56(\mathrm{DP}=6,31)$ & $27,80(\mathrm{DP}=6,16)$ & 0,693 \\
Ambiência & $22,98(\mathrm{DP}=4,36)$ & $23,30(\mathrm{DP}=3,63)$ & $23,15(\mathrm{DP}=3,97)$ & 0,698 \\
\hline
\end{tabular}

Nota. Tabela com médias e desvios-padrão em duas dimensões avaliadas. AA= discentes ingressantes no ensino superior por Ações Afirmativas; $\mathrm{AC}=$ discentes ingressantes por Ampla Concorrência.

* Teste $t$ de Student com 005 de significância (probabilidade de erro de 5\%).

Fonte: Autores.

As médias das dimensões pedagógica e de ambiência variaram de 27,80 (DP=6,16) e 23,15 (DP=3,97), respectivamente, revelando uma relativa satisfação com o ERE, porém evidencia que alguns aspectos devem ser melhorados. Não foram verificadas diferenças significativas entre as pontuações obtidas pelos ingressos por AA e AC.

Na Tabela 3 encontram-se as medianas e intervalo interquartil (p25-p75) das pontuações dos itens sobre a percepção dos estudantes obtidas por meio da escala Likert aplicada no segundo momento do estudo.

Tabela 3. Percepção dos estudantes sobre o Ensino Remoto Emergencial no primeiro semestre de implementação. Goiânia, Goiás, Brasil, 2020.

\begin{tabular}{|c|c|c|c|c|c|c|}
\hline \multirow[t]{2}{*}{ Questões } & \multirow{2}{*}{$\begin{array}{l}\text { Mediana } \\
(\text { p25-75) }\end{array}$} & \multicolumn{4}{|c|}{ Escala } & \multirow{2}{*}{$\begin{array}{l}\text { Valor- } \\
\mathbf{p}^{*}\end{array}$} \\
\hline & & $\begin{array}{c}1 \\
\mathbf{n}(\%)\end{array}$ & $\begin{array}{c}2 \\
\mathbf{n}(\%)\end{array}$ & $\begin{array}{c}3 \\
\mathbf{n}(\%)\end{array}$ & $\begin{array}{c}4 \\
\mathbf{n}(\%)\end{array}$ & \\
\hline $\begin{array}{l}\text { 1. Considero que o Ensino Remoto Emergencial (ERE) } \\
\text { foi necessário diante da pandemia por COVID-19. }\end{array}$ & $4(4-4)$ & 0 & $3(3,2)$ & $\begin{array}{l}17 \\
(18,3)\end{array}$ & $\begin{array}{l}73 \\
(78,5)\end{array}$ & $<0,001$ \\
\hline $\begin{array}{l}\text { 2. O meu desempenho/interesse durante o ERE foi } \\
\text { satisfatório para o meu aprendizado. }\end{array}$ & $3(3-4)$ & $3(3,2)$ & $19(20,4)$ & $\begin{array}{l}45 \\
(48,4)\end{array}$ & $26(28)$ & $<0,001$ \\
\hline $\begin{array}{l}\text { 3. Houve uma boa interação com os professores no } \\
\text { decorrer das disciplinas. }\end{array}$ & $3(3-3)$ & $2(2,2)$ & $18(19,4)$ & $\begin{array}{l}51 \\
(54,8)\end{array}$ & $\begin{array}{l}22 \\
(23,7)\end{array}$ & $<0,001$ \\
\hline $\begin{array}{l}\text { 4. A interação com os professores e colegas de turma } \\
\text { durante o ERE influenciou positivamente no meu } \\
\text { estado emocional. }\end{array}$ & $3(2-4)$ & $4(4,3)$ & $28(30,1)$ & $\begin{array}{l}32 \\
(34,4)\end{array}$ & $\begin{array}{l}29 \\
(31,2)\end{array}$ & $<0,001$ \\
\hline $\begin{array}{l}\text { 5. O ERE contribuiu de forma negativa para a minha } \\
\text { saúde mental. }\end{array}$ & $3(2-4)$ & $10(10,8)$ & $33(35,5)$ & $\begin{array}{l}25 \\
(26,9)\end{array}$ & $\begin{array}{l}25 \\
(26,9)\end{array}$ & 0,008 \\
\hline $\begin{array}{l}\text { 6. O meu ambiente de estudo em casa apresentou } \\
\text { qualidade satisfatória para o desenvolvimento das } \\
\text { atividades remotas. }\end{array}$ & $3(2-4)$ & $8(8,6)$ & $23(24,7)$ & $\begin{array}{l}33 \\
(35,5)\end{array}$ & $\begin{array}{l}29 \\
(31,2)\end{array}$ & 0,001 \\
\hline $\begin{array}{l}\text { 7. Eu precisei de suporte da Universidade para uso das } \\
\text { Tecnologias Digitais de Informação e Comunicação } \\
\text { (TDIC). }\end{array}$ & $1(1-2,5)$ & $59(63,4)$ & $11(11,8)$ & $\begin{array}{l}11 \\
(11,8)\end{array}$ & $\begin{array}{l}12 \\
(12,9)\end{array}$ & $<0,001$ \\
\hline $\begin{array}{l}\text { 8. O suporte oferecido pela Universidade para o uso das } \\
\text { TDIC (treinamento, suporte/assistência...) foi } \\
\text { satisfatório. }\end{array}$ & $3(3-4)$ & $2(2,2)$ & $14(15,1)$ & $\begin{array}{l}48 \\
(51,6)\end{array}$ & $\begin{array}{l}29 \\
(31,2)\end{array}$ & $<0,001$ \\
\hline $\begin{array}{l}\text { 9. Fiquei satisfeito com as aulas teóricas realizadas de } \\
\text { forma remota. }\end{array}$ & $3(2-4)$ & $5(5,4)$ & $23(24,7)$ & $\begin{array}{l}38 \\
(40,9)\end{array}$ & 27 (29) & $<0,001$ \\
\hline $\begin{array}{l}\text { 10. Fiquei satisfeito com as aulas práticas realizadas de } \\
\text { forma remota. }\end{array}$ & $2(1-3)$ & $35(37,6)$ & $29(31,2)$ & $\begin{array}{l}20 \\
(21,5)\end{array}$ & $9(9,7)$ & 0,001 \\
\hline
\end{tabular}


11. Meus insumos tecnológicos

$4(3-4) \quad 1(1,1) \quad 17(18,3) \quad 28$

47

$<0,001$

(equipamentos/conexão) foram suficientes/adequados

$(30,1)$

$(50,5)$

para a realização do ensino remoto de forma

satisfatória.

12. Os conteúdos das disciplinas foram abordados de

$\begin{array}{llllll}3(2-3) & 8(8,6) & 33(35,5) & 39 & 13(14) & <\mathbf{0 , 0 0 1} \\ & & & (41,9) & & \\ 3(2-3) & 10(10,8) & 25(26,9) & \begin{array}{l}38 \\ (40,9)\end{array} & \begin{array}{l}20 \\ (21,5)\end{array} & \mathbf{0 , 0 0 1} \\ & & & & \\ 3(2-4) & 6(6,5) & 18(19,4) & 40(43) & \begin{array}{l}29 \\ (31,2)\end{array} & <\mathbf{0 , 0 0 1} \\ & & & & & \\ 3(3-4) & 4(4,3) & 15(16,1) & 49 & 25 & <\mathbf{0 , 0 0 1} \\ & & & \begin{array}{l}52,7) \\ (26,9)\end{array} & \\ 3(2-4) & 14(15,1) & 20(21,5) & 34 & \begin{array}{l}25 \\ (36,6)\end{array} & \mathbf{0 , 0 2 6} \\ & & & 26,9) & \\ 3(2-3) & 3(3,2) & 36(38,7) & \begin{array}{l}39 \\ (41,9)\end{array} & \begin{array}{l}15 \\ (16,1)\end{array} & <\mathbf{0 , 0 0 1} \\ 3(2-3) & 12(12,9) & 24(25,8) & \begin{array}{l}35 \\ (37,6)\end{array} & \begin{array}{l}22 \\ (23,7)\end{array} & \mathbf{0 , 0 0 9} \\ & & & (37)\end{array}$

forma dinâmica, criativa e estimularam o interesse.

13. O material de apoio pedagógico fornecido na

disciplina foi suficiente para a aprendizagem.

14. O suporte oferecido pelos

professores/instrutores/monitores se deu de forma

satisfatória.

15. Os processos de avaliação da aprendizagem

realizados nas disciplinas foram satisfatórios.

16. O prazo para o desenvolvimento das atividades

avaliativas e complementares foi suficiente.

17. O feedback das atividades avaliativas e

complementares foi satisfatório.

18. No geral estou satisfeito com o ERE.

$(37,6)$

$(23,7)$

Nota. Tabela com percepções dos estudantes acerca da experiência vivenciada durante o primeiro semestre de implantação do ERE.

*Qui-quadrado de independência com 005 de significância (probabilidade de erro de 5\%).

Fonte: Autores.

A melhor dinâmica para aulas teóricas $(n=57 ; 61,3 \%)$ e práticas $(n=36 ; 38,7 \%)$ foi a organização das disciplinas em aulas síncronas e assíncronas. A distribuição da carga horária das disciplinas entre as aulas síncronas e assíncronas foi considerada adequada pela maior parte dos discentes $(n=37 ; 39,8 \%)$, seguida da sugestão de reduzir a carga horária das aulas síncronas e aumentar das assíncronas $(\mathrm{n}=36 ; 38,7 \%)$. A plataforma GSuite $(\mathrm{n}=78 ; 83,9 \%)$ foi aquela que possibilitou o melhor ambiente de aprendizagem segundo os estudantes, sem diferenças entre AA e AC (Tabela 4). 
Tabela 4. Avaliação do Ensino Remoto Emergencial no primeiro semestre de sua implantação. Goiânia, Goiás, Brasil, 2021.

\begin{tabular}{|c|c|c|c|c|c|}
\hline Questionamentos & Respostas & $\begin{array}{c}\mathrm{AA} \\
\mathrm{n}=\mathbf{4 3} \\
(\mathbf{4 6 , 2} \%)\end{array}$ & $\begin{array}{c}\mathrm{AC} \\
\mathbf{n}=\mathbf{5 0} \\
(\mathbf{5 3}, \mathbf{8 \%})\end{array}$ & $\begin{array}{c}\text { Total } \\
\mathbf{n = 9 3} \\
(\mathbf{1 0 0 \%})\end{array}$ & Valor-p* \\
\hline $\begin{array}{l}\text { Melhor dinâmica para } \\
\text { as aulas teóricas }\end{array}$ & $\begin{array}{c}\text { Síncrona } \\
\text { Assíncrona } \\
\text { Síncrona + Assíncrona }\end{array}$ & $\begin{array}{c}6(6,5 \%) \\
11(11,8 \%) \\
26(28 \%)\end{array}$ & $\begin{array}{c}6(6,5 \%) \\
13(14 \%) \\
31(33,3 \%)\end{array}$ & $\begin{array}{l}12(12,9 \%) \\
24(25,8 \%) \\
57(61,3 \%)\end{array}$ & 0,961 \\
\hline $\begin{array}{c}\text { Melhor dinâmica para } \\
\text { as aulas práticas }\end{array}$ & $\begin{array}{c}\text { Síncrona } \\
\text { Assíncrona } \\
\text { Síncrona + Assíncrona }\end{array}$ & $\begin{array}{l}12(12,9 \%) \\
15(16,1 \%) \\
16(17,2 \%)\end{array}$ & $\begin{array}{l}17(18,3 \%) \\
13(14 \%) \\
20(21,5 \%)\end{array}$ & $\begin{array}{l}29(31,2 \%) \\
28(30,1 \%) \\
36(38,7 \%)\end{array}$ & 0,629 \\
\hline $\begin{array}{c}\text { Distribuição da carga } \\
\text { horária das disciplinas } \\
\text { em atividades síncronas } \\
\text { e assíncronas }\end{array}$ & $\begin{array}{c}\text { Adequada } \\
\text { Reduzir síncrona e aumentar } \\
\text { assíncrona } \\
\text { Aumentar síncrona e reduzir } \\
\text { assíncrona }\end{array}$ & $\begin{array}{c}16(17,2 \%) \\
14(15,1 \%) \\
13(14 \%)\end{array}$ & $\begin{array}{c}21(22,6 \%) \\
22(23,7 \%) \\
7(7,5 \%)\end{array}$ & $\begin{array}{l}37(39,8 \%) \\
36(38,7 \%) \\
20(21,5 \%)\end{array}$ & 0,154 \\
\hline $\begin{array}{c}\text { Plataforma de TDIC } \\
\text { que possibilitou o } \\
\text { melhor ambiente de } \\
\text { ensino / aprendizagem }\end{array}$ & $\begin{array}{c}\text { GSuite (Google Meet, Classroom, etc.) } \\
\text { Moodle } \\
\text { SIGAA ou RNP }\end{array}$ & $\begin{array}{c}38(40,9 \%) \\
4(4,3 \%) \\
1(1,1 \%)\end{array}$ & $\begin{array}{l}40(43 \%) \\
8(8,6 \%) \\
2(2,2 \%)\end{array}$ & $\begin{array}{c}78(83,9 \%) \\
12(12,9 \%) \\
3(3,2 \%)\end{array}$ & 0,549 \\
\hline
\end{tabular}

Nota. Tabela com avaliação dos estudantes acerca da experiência vivenciada durante o primeiro semestre de implantação do ERE. SIGAA: Sistema Integrado de Gestão de Atividades Acadêmicas. RNP: Rede Nacional de Ensino e Pesquisa.

*Qui-quadrado de independência com 005 de significância (probabilidade de erro de 5\%).

Fonte: Autores.

\section{Discussão}

A necessidade da operacionalização do ERE trouxe a preocupação em garantir um ensino de qualidade com equidade. Apesar das diferenças significativas verificadas na primeira etapa do estudo no tipo e qualidade da internet, no ambiente de estudos e nos equipamentos utilizados pelos discentes AA e AC, a percepção dos alunos após a implantação do ERE não apresentou diferenças entre as categorias de ingresso. Isso sinaliza que as ações desenvolvidas pela universidade e pelo Curso de Nutrição foram importantes para mitigar os impactos referentes às condições de conectividade e de ambiência no aprendizado desses alunos, principalmente naqueles em maior vulnerabilidade social. Nesse sentido, os resultados do presente trabalho reiteram os estudos com ERE na Faculdade de Medicina da Universidade Estadual de Campinas/UNICAMP em que os autores concluíram que caracterizar os estudantes quanto à dificuldade de acesso, a escuta atenta e políticas internas de empréstimos e doação de equipamentos reduzem a iniquidade em relação ao acesso às plataformas remotas, de forma a evitar que o ERE seja um fator de desigualdade no processo ensino-aprendizagem (Appenzeller et al., 2020).

Os nossos resultados refletem a atual situação do país em que cerca de 78,3\% dos brasileiros estão conectados, o que possibilita ao Brasil ocupar a $5^{\text {a }}$ posição no ranking de países em população online (Menezes, 2021). Entretanto, a qualidade dessa conectividade é precária, cara e sem equidade. O departamento gestor da internet no Brasil, que monitora a adoção de tecnologias de informação há 15 anos, mostra que 15 milhões de pessoas, especialmente a população mais pobre, só utilizam internet pelo celular. Segundo este departamento, as desigualdades de conexão à rede são classificadas em dois níveis: 1) ter ou não acesso e 2) acessar, mas com diversas limitações. Dentre essas está a localização do indivíduo, uma vez que a dificuldade de acesso ocorre em bairros periféricos ou até mesmo regiões muito adensadas. Em nossos resultados, a maioria dos estudantes possui acesso, mas o fator preocupante foi na limitação e na qualidade do acesso, pelo pacote de dados disponível que foi agravado pelo caso de compartilhamento dos equipamentos, dificultando acompanhar as aulas síncronas. Estes resultados corroboram com Pérez-López, Atochero e Rivero (2021). 
Várias universidades adotaram recentemente políticas de democratização de acesso ao ensino superior, as AA (Brasil, 2012). O ERE evidenciou a desigualdade existente entre distintas classes sociais dentro destas instituições. Muitos estudantes com dificuldades de acesso ou sem acesso à internet não conseguem conectar-se às plataformas virtuais de ensino (Souza \& Miranda, 2020) e tiveram que optar por não se matricular no curso neste semestre ou ficar com ensino falho.

Estudo sobre os efeitos do ERE na prática educacional do estado do Rio Grande do Sul relatou evidente custo social pelo fechamento das escolas (Lopes, 2020). O autor revelou preocupação com os estudantes sem acesso à internet ou com recursos tecnológicos insuficientes para permitir o desempenho igualitário entre todos. As perspectivas de existência de perdas pedagógicas e abismo social e intelectual construído entre os estudantes após o período de isolamento social são evidentes. Nossos resultados também demonstraram perdas dos alunos de AA em relação aos alunos de AC.

Políticas públicas educacionais não podem se restringir ao acesso ao ensino superior. A efetiva democratização do ensino superior deve considerar o acesso, a permanência e o êxito são ações que devem ser indissociáveis. Afinal, o acesso isolado pode mascarar as desigualdades educacionais. Assim, torna-se fundamental a análise das políticas de ações afirmativas baseada também nos outros dois aspectos: sucesso e permanência dos estudantes (Silva \& Oliveira, 2019).

Durante os primeiros meses da pandemia, a universidade promoveu debates, cursos e eventos online para refletir sobre o novo cenário e as necessidades do ERE para a continuidade do ensino. Tal fato culminou em iniciativas de apoio aos estudantes, professores, técnicos e gestores. No âmbito da assistência estudantil, foi proposto o Plano de Conectividade, voltado para estudantes de renda per capita menor que 1,5 salário-mínimo, com a finalidade de instituir a política de empréstimo de equipamentos de informática para este segmento de estudantes. Foi realizado um repasse financeiro para o pagamento de pacote de dados móveis ou para a aquisição de equipamento de informática (Universidade Federal de Goiás [UFG], 2020d).

Além disso, foi criado o site UFG em Casa (https://ufgemcasa.ufg.br/, recuperado em 22 de maio de 2021) que orientou a comunidade universitária sobre os procedimentos necessários para o uso de meios digitais de forma não presencial. Especificamente para os estudantes, o site ofereceu o ícone perguntas frequentes sobre o reinício das aulas que tratavam de normativas e orientações da gestão acadêmica relacionadas às matrículas, disciplinas disponibilizadas, estágio obrigatório e não obrigatório. Também estavam disponíveis tutoriais sobre como usar as ferramentas institucionais, cursos, módulos e ações de formação para o estudante, informações sobre como se portar nos diálogos virtuais e no uso de informações remotas, orientações sobre o atendimento psicológico online, além de Portarias, Resoluções e Infográficos sobre o plano de conectividade da UFG. Já o NDE da FANUT induziu discussões junto ao corpo docente, estudantes e servidores a fim de tratar das especificidades do curso, principalmente quanto às disciplinas que estavam em andamento, a reorganização das aulas práticas que ocorriam em serviços de saúde e laboratórios, e os estágios curriculares obrigatórios.

Essas iniciativas foram de encontro à preocupação institucional em evitar a exclusão de estudantes e em garantir o acesso e a qualidade do ensino. Neste estudo, 2,9\% dos estudantes referiram precisar de suporte da universidade para uso das TDIC. Segundo estudo de Valente, Moraes, Sanchez, Souza e Pacheco (2020), a escolha em participar do ERE não é uma alternativa do aluno e sim a única alternativa, tendo a compreensão que esse formato é emergencial e não uma substituição em definitivo das aulas presenciais, com todos outros recursos, incluindo laboratórios e campos de prática, além da singularidade de interação diária com os estudantes. Embora se compreenda que muita atividade aplicada no ERE se tornará, no póspandemia, parte das rotinas acadêmicas.

Apesar das limitações do ERE, principalmente do ponto de vista da vivência, socialização e interação (Rodini, Pedro \& Duarte, 2020), e da relativa satisfação dos estudantes quanto à expectativa relacionada aos aspectos pedagógicos e ambiência observados no primeiro momento do presente estudo, essa percepção continuou. Após cursar um semestre inteiro na modalidade ERE, na maioria esteve inclinada a concordar ou concordou que, em geral, estava satisfeita com o ERE. No 
entanto, a ampla maioria discordou ou mostrou-se inclinada a discordar quanto à satisfação das aulas práticas realizadas de forma remota. Sendo assim, apesar de todo o aparato para o acesso às TDIC oferecido pela Universidade, o aprendizado em serviço é uma etapa necessária ao processo de aquisição de habilidades e competências que antecedem a formação do profissional de saúde (Torres et al., 2021).

A fim de preencher essa lacuna de falta de aulas, a Universidade autorizou a realização de aulas práticas emergenciais (Universidade Federal de Goiás [UFG], 2020e). Diversos fatores contribuíram para a reflexão sobre o processo de trabalho/aulas práticas em saúde na pandemia, como: as limitações de espaço físico necessárias ao distanciamento social no serviço de saúde, escassez no mercado de equipamentos de equipamentos de proteção individual e ausência de vacinação universal que garantisse a segurança dos estudantes em serviço. Diante desse cenário, o uso das TDIC oportunizou inovações pedagógicas para o desenvolvimento de novos formatos de aula prática, embora não substituíssem a vivência no serviço de saúde. Desse modo, foi possível realizar assistência nutricional por videoconferência (Conselho Federal de Nutricionistas [CFN], 2020), visita virtual aos serviços de saúde, relatos de experiências profissionais e observação de experimentos em laboratório por meio de videoaulas.

Apesar dos esforços, parte dos estudantes referiram algum grau de insatisfação quanto ao ERE $(38,7 \% ; \mathrm{n}=36)$ e efeito negativo do ERE na saúde mental $(46,3 \% ; \mathrm{n}$ = 43). Dados com impacto semelhante foram observados em 1182 estudantes de diferentes faixas etárias na Índia, indicando que hábitos de sono, rotinas diárias de condicionamento físico e a interação social no ERE afetaram significativamente suas condições de saúde (Chaturvedi, Vishwakarma, \& Singh, 2020). O medo da contaminação e as medidas de distanciamento social somadas às incertezas quanto ao futuro de sua formação (Rodrigues et al., 2020), além da crise econômica, das alterações na dinâmica familiar, do adoecimento e da perda de entes queridos também repercutem sobre a saúde mental dos estudantes e impactam as rotinas acadêmicas (Gundim et al., 2021). Nesse sentido, a escuta ativa aos resultados do presente estudo são importantes para oferecer um ERE que favoreça o aprendizado, bem-estar e interação dos estudantes.

Nesse sentido, a avaliação da distribuição e a carga horária das aulas síncronas e assíncronas e as plataformas de aprendizagem trouxeram informações relevantes para melhorar essa interação, minimizar o isolamento social e promover uma melhor experiência de estudo. Tanto os estudantes ingressos por AC e AA reconheceram a necessidade de distribuição das aulas em síncronas e assíncronas, contudo, parte deles sugeriram o aumento da carga horária das aulas assíncronas. Essa sugestão expõe o anseio de solucionar as dificuldades na ambiência e conectividade e oportuniza modificar a dinâmica de interação entre professores e estudantes.

A interação é um indicador de qualidade da educação superior e deve ser permanente e continuada em diferentes espaços geográficos e tempos (Brasil, 2015). Nunes, Pereira \& Brasileiro (2018) observaram que o nível de interação síncrona varia de baixo a médio na percepção da maioria dos estudantes e baixa interação para docentes. Já para o nível de interação assíncrona observaram percepção mediana na avaliação de estudantes e docentes, tendo o e-mail e o fórum como tecnologias assíncronas que promoveram maior interação. Assim, percebe-se que para o ERE a atividade assíncrona deve ser vista como um importante meio de aprendizado que pode oportunizar ao estudante o acesso ao conteúdo e o desenvolvimento de habilidades de acordo com a sua disponibilidade de tempo, ambiência e conectividade.

\section{Conclusão}

A política das AA é uma política pública que busca elevar os níveis educacionais, combater as iniquidades e melhorar as condições de vida daqueles excluídos socialmente. Com a pandemia do Novo Coronavirus desvelou dificuldades a serem enfrentadas no ensino superior, principalmente entre os estudantes das AA. 
Com este trabalho percebeu-se as dificuldades enfrentadas na gestão do ERE, bem como os problemas defrontados por muitos estudantes, especialmente aqueles ingressos no ensino superior por AA, devido à falta de recursos tecnológicos e ambiência demandados neste cenário. Entretanto, com o auxílio institucional, o Ensino Remoto Emergencial, na percepção dos estudantes, atendeu, neste primeiro semestre de sua implantação, as exigências educacionais advindas da pandemia ocasionada pelo Sars-CoV-2.

Os resultados da pesquisa evidenciam a necessidade de um acompanhamento sistemático e atento da gestão do curso de Nutrição, e extrapolando os resultados, para todos os Cursos de ensino superior das universidades federais no Brasil, bem como o envolvimento de professores e estudantes na discussão de estratégias para superação das dificuldades.

No cenário atual, a preocupação central não é só a permanência do estudante, mas também a qualidade do ensino e uma formação que responda às necessidades de saúde e nutrição da população. Neste sentido, políticas públicas de apoio as universidades federais são essenciais nesse processo. A dimensão das consequências pós-pandemia em todos os setores, inclusive na educação e principalmente no grupo de estudantes ingressos nas AA, são desconhecidas.

Pesquisas futuras sobre a influência do ERE na formação do profissional nutricionista, bem como o acompanhamento do egresso por parte da gestão do curso no período pós-pandemia possibilitarão uma avaliação mais ampla dessa forma de ensino.

\section{Referências}

Al Samaraee, A. (2020). The impact of the COVID-19 pandemic on medical education. British Journal of Hospital Medicine, 81(7), 1-4. https://doi.org/10.12968/ hmed.2020.0191

Appenzeller, S., Menezes, F. H., Santos, G. G., Padilha, R. F., Graça, H. S., \& Bragança, J. F. (2020). Novos Tempos, Novos Desafios: Estratégias para Equidade de Acesso ao Ensino Remoto Emergencial. Revista Brasileira de Educação Médica, 44(1), e0155. https://doi.org/10.1590/1981-5271v44.supl.120200420

Brasil. (2012). Lei $N^{o} 12.711$, de 29 de agosto de 2012. Dispõe sobre o ingresso nas universidades federais e nas instituições federais de ensino técnico de nível médio e dá outras providências. http://portal.mec.gov.br/cotas/docs/lei_12711_29_08_2012.pdf

Brasil. Ministério da Educação. Instituto Nacional de Estudos e Pesquisas Educacionais Anísio Teixeira - Inep. (2015). Instrumento de Avaliação de Cursos de Graduação presencial e a distância. http://download.inep.gov.br/educacao_superior/avaliacao_cursos_graduacao/instru mentos/2015/instrumento_avaliacao_cursos_graduacao_presencial_distancia.pdf

Cardoso, J. A. (2020). Pandemia e o regime especial de aulas não presenciais: analisando o modelo da Secretaria de Educação do Estado de Goiás - SEDUC. Revista Aproximação, 2(5), 33-45.

Chaturvedi, K., Vishwakarma, D. K., \& Singh, N. (2021). COVID-19 and its impact on education, social life and mental health of students: A survey. Children and Youth Services Review, 121, 105866. https://doi.org/10.1016/j.childyouth.2020.105866

Conselho Federal de Nutricionistas (2020). Resolução do Conselho Federal de Nutricionistas $n^{o}$ 646, de 18 de março de 2020 . Suspende até o dia 31 de agosto de 2020 o disposto no artigo 36 da Resolução do Conselho Federal de Nutricionistas n ${ }^{\circ} 599$, de 25 de fevereiro de 2018 , que aprova o Código de Ética e de Conduta dos Nutricionistas.

Fórum Nacional de Pró- Reitores de Assuntos Comunitários e Estudantis (2019). V Pesquisa Nacional de Perfil Socioeconômico e Cultural dos (as) Graduandos (as) das IFES - 2018. Associação Nacional dos Dirigentes das Instituições Federais de Ensino Superior. https://www.andifes.org.br/wpcontent/uploads/2019/05/V-Pesquisa-Nacional-de-Perfil-Socioeconomico-e-Cultural-dos-as-Graduandos-as-das-IFES-2018.pdf

Gundim, V. A., Encarnação, J. P., Santos, F. C., Santos, J. E. dos, Vasconcellos, E. A., \& Souza, R. C. de. (2021). Saúde mental de estudantes universitários durante a pandemia de COVID-19. Revista Baiana de Enfermagem, 35, e37293. http://dx.doi.org/10.18471/rbe.v35.37293

Hair, J. F. Jr., Anderson, R. E., Tatham, R. L., \& Black, W. C. (2002). Multivariate data analysis (5a ed.) Englewood Cliffs, NJ: Prentice Hall.

Joaquin, J. J. B., Biana, H. T., \& Dacela, M. A. (2020). The Philippine Higher Education Sector in the Time of COVID-19. Frontiers in Education, 5, 1-6. https://doi.org/10.3389/feduc.2020.576371

Lopes, P. C. A. B. (2020). A Covid-19, o retorno às aulas e o custo social do fechamento das escolas - o que pode ser feito? Revista Educação Pública, 20(29), 1-8. https://educacaopublica.cecierj.edu.br/artigos/20/29/a-covid-19-o-retorno-as-aulas-e-o-custo-social-do-fechamento-das-escolas-o-quepode-ser-feito

Menezes, V. (2021, abril 26). Brasil está entre os cinco países do mundo que mais usam internet. Entrevista no evento da Organização para a Cooperação e Desenvolvimento Econômico. https://www.gov.br/pt-br/noticias/transito-e-transportes/2021/04/brasil-esta-entre-os-cinco-paises-do-mundo-que-mais-usaminternet\#: :text=Com\%2078\%2C3\%25\%20de\%20brasileiros,fibras\%20\%C3\%B3pticas\%20\%C3\%A0s\%20redes\%20nacionais. 
Moehlecke, S. (2002). Ação afirmativa: história e debates no Brasil. Cadernos de Pesquisa, 117, 197-217.

Nunes, E. B. L. de L. P., Pereira, I. C. A. \& Brasileiro, T. S. A. (2018). A interação como indicador de qualidade na avaliação da educação a distância: um estudo de caso com docentes, tutores e discentes. Avaliação: Revista da Avaliação da Educação Superior, $23(3)$, 869-887. https://doi.org/10.1590/S1414-40772018000300017

Pérez-López, E., Atochero, A. V., \& Rivero, S. C. (2021). Educación a distancia en tiempos de COVID-19: Análisis desde la perspectiva de los estudiantes universitários. RIED. Revista Iberoamericana de Educación a Distancia, 24(1), 331-350. http://dx.doi.org/10.5944/ried.24.1.27855

Petretto, D. R., Masala, I., \& Masala, C. (2020). Special educational needs, distance learning, inclusion and COVID-19. Education Sciences, 10(6), 154. https://doi.org/10.3390/educsci10060154

Rajab, M. H., Gazal, A. M., \& Alkattan, K. (2020). Challenges to Online Medical Education During the COVID-19 Pandemic. Cureus, 12(7), e8966. https://doi.org/10.7759/cureus.8966

Rondini, C. A., Pedro, K. M., \& Duarte, C. dos S. (2020). Pandemia do covid-19 e o ensino remoto emergencial: mudanças na práxis docente. Educação, 10(1), 41-57. https://doi.org/10.17564/2316-3828.2020v10n1p41-57

Sandhu, P., \& de Wolf, M. (2020). The impact of COVID-19 on the undergraduate medical curriculum. Medical Education Online, 25(1), 1764740. https://doi.org/101080/10872981.2020.1764740

Silva, I. Jr., \& Oliveira, A. C. D. C. (2019). Igualdade de oportunidades no ensino superior brasileiro: ações afirmativas e indicadores de avaliação de políticas como mediadores do sucesso escolar. Cadernos de Pesquisa: Pensamento Educacional, 14(36), 186-201.

Sindiani, A. M., Obeidat, N., Alshdaifat, E., Elsalem, L., Alwani, M. M., Rawashdeh, H., Fares, A. S., Alalawne, T., \& Tawalbeh, L. I. (2020). Distance education during the COVID-19 outbreak: A cross-sectional study among medical students in North of Jordan. Annals of Medicine and Surgery, 59, 186-194. https://doi.org/10.1016/j.amsu.2020.09.036

Souza, D. G., \& Miranda, J. C. (2020). Desafios da implementação do ensino remoto. Boletim da Conjuntura, 4(11). http://doi.org/10.5281/zenodo.4252805

Torres, F. J. R., Correa, A. C de S., Freitas, C. A. S. L., Santos, R. L. dos, Rodrigues, A. R. M., \& Dias, M. S. de A. (2021). Programa de Educação pelo Trabalho e Saúde (PET - Saúde) Interprofissionalidade: aproximações e distanciamentos com as definições de Educação Interprofissional. Pesquisa, Sociedade e Desenvolvimento,10(1), e40610111862. https://rsdjournal.org/index.php/rsd/article/view/11862

Universidade Federal de Goiás. (2020a). Resolução CONSUNI $n^{o}$ 34/2020. Aprova a Instrução Normativa CONSUNI No 01/2020 que dispõe sobre procedimentos relativos a todas as atividades escolares da educação básica e acadêmicas da graduação no modo de ensino remoto.

Universidade Federal de Goiás. (2020b). Resolução CONSUNI $n^{\circ}$ 33/2020. Franqueia regras do Regulamento Geral dos Cursos de Graduação - RGCG, aprovado pela Resolução CEPEC N ${ }^{\circ} 1557$, de 1\%12/2017, enquanto durar a situação de pandemia do Novo Coronavírus e até nova deliberação.

Universidade Federal de Goiás (2020c). Resolução CONSUNI $n^{\circ}$ 35/2020. Aprova o novo Calendário Acadêmico da Universidade Federal de Goiás, Regional Goiânia e Regional Goiás, para o ano letivo de 2020, revogando as resoluções CEPEC No 1663 e 1664, de 29 de novembro de 2019.

Universidade Federal de Goiás (2020d). Portaria SEI $n^{\circ}$ 26/2020. Dispõe sobre o atendimento de estudantes com dificuldades advindas ou agravadas no contexto de crise provocada pela COVID-19, a serem atendidos com repasse financeiro único, em caráter emergencial, destinado a contribuir com a aquisição de equipamentos de proteção individual (EPI), como parte de procedimentos de segurança para a retomada presencial das turmas, parcialmente ou integralmente suspensas, na forma de Atividades Práticas Emergenciais.

Universidade Federal de Goiás (2020e). Resolução CONSUNI $n^{\circ}$ 61/2020. Aprova a Instrução Normativa CONSUNI N ${ }^{\circ}$ 02/2020, que dispõe sobre os procedimentos relativos à retomada presencial das turmas parcialmente ou integralmente suspensas na forma de Atividades Práticas Emergenciais (APEs) e dá outras providências.

Valente, G. S. C., Moraes, É. B., Sanchez, M. C. O., Souza, D. F., \& Pacheco, M. C. M. D. (2020). O ensino remoto frente às exigências do contexto de pandemia: Reflexões sobre a prática docente. Research, Society and Development, 9(9), e843998153. http://dx.doi.org/10.33448/rsd-v9i9.8153

Tanik-Önal, N., \& Önal, N. (2020). Teaching Science through Distance Education during the COVID-19 Pandemic. International Online Journal of Education and Teaching, 7(4), 1898-1911. 